Proceedings

\title{
Towards Information Ecosystem for Urban Planning-The Application of Video Data ${ }^{\dagger}$
}

\author{
Longmei Han ${ }^{1}$, Linbo Qing ${ }^{2, *}$,Yonghong Peng ${ }^{3}$ and Xiaohai $\mathrm{He}^{2}$ \\ 1 Chengdu Institute of Planning \& Design, No. 399 Fucheng Avenue, 610041 Chengdu, China; \\ hanlongmei@163.com \\ 2 College of Electronics and Information Engineering, Sichuan University, No.24 South Section 1, Yihuan \\ Road, 610065 Chengdu, China; qing_lb@scu.edu.cn(L.Q.);hxh@scu.edu.cn (X.H.) \\ 3 Faculty of Computer Science, University of Sunderland, St Peters Campus, SR6 0DD, UK; \\ Yonghong.Peng@sunderland.ac.uk \\ * Correspondence: qing_lb@scu.edu.cn; Tel.: +86-028-8546-3466 \\ + Presented at the IS4SI 2017 Summit DIGITALISATION FOR A SUSTAINABLE SOCIETY, Gothenburg, \\ Sweden, 12-16 June 2017.
}

Published: 9 June 2017

\begin{abstract}
In this study we present a new ecosystem for urban planning which highly depended on a variety of basic information. From information ecology view, the urban planning process could be seen as an ecological chain with the conversion of "survey-analysis-planning-implementation". Over decades' urban researchers usually focus on the conversion of "planning" and "implementation", while less effort has been made in the "survey" and "analysis" part. Most of works only collect limited data from which little information can be obtained describing human activity. In order to improve the information ecosystem for urban planning, we first introduces "Street Vibrancy Assessment based on Video Data" into urban planning, focusing on the understanding of human activity. Compared with other data, video data is relevant more accurate and more reliable at micro-scale. Based on video data, an urban planning case is designed and discussed. In such case, the human mobility and activity parameters based on video data are analyzed and fed into a specific calculation model to get the external representation of street vibrancy. The correlation between the external representation and the constituent elements of the street vibrancy are exploited afterward for the assessment. Finally the assessment results can be used to guide the "planning" and "implementation" of the urban planning ecosystem.
\end{abstract}

Keywords: urban planning; street vibrancy; mobility and activity; video data

\section{Introduction}

Urban planning is a subject which is highly relying on basic information. The main typical urban planning process consists of Survey, Analysis, Planning and Implementation. Over decades' urban researchers usually focus on the process of "Planning" and "Implementation", while less effort has been made in the "Survey" and "Analysis" part. In the meantime, most of the work only deal with one of the processes with other ones unconcerned. How to deal with the urban planning task as a whole and achieve global optimization is crucial for modern urban planning. Information ecology is the study of interrelations among episodes of information conversion and their environment for better performance. Information ecology has been widely applied in the field of E-Commerce and Education to achieve global optimization, but has not yet been adopted in the field of urban planning. It is of great significance to examine the information unbalance in urban planning from the perspective of information ecology, which is helpful to the extensive collection and efficient use of information, and to accomplish more scientific, rigorous and effective planning. 
This work will adopt the ecological methodology to guide the research on urban planning. Specifically, the Information Eco-System of planning will be modeled and analyzed, and the special attention will be paid to the information unbalance problem of urban planning. Finally, a solution will be given with additional discussion.

\section{The problem of Urban Planning Information Eco-System}

\subsection{Information Eco-system Model of Urban Planning}

The whole urban planning process as an ecological system is referred to the conversion of" survey-analysis-planning-implementation" (Figure1). In the beginning the information of objects in urban (e.g., residents, roads, streets, lands et al.) are collected by surveying and investigating. Then selected information are analyzed to form urban knowledge. With the support of the urban knowledge, researchers and managers could make a proper planning. The planning strategy would guide the urban construction and management, which is called planning implementation. Finally, the planning would affect the objects in urban. More walkable streets, more convenient instruction, more efficient transportation would make better urban life. Unfortunately, some important parts are missing in urban planning system. Consequently, the lack of information collection break the balance of the whole urban planning ecological chain and make the planning less successful.

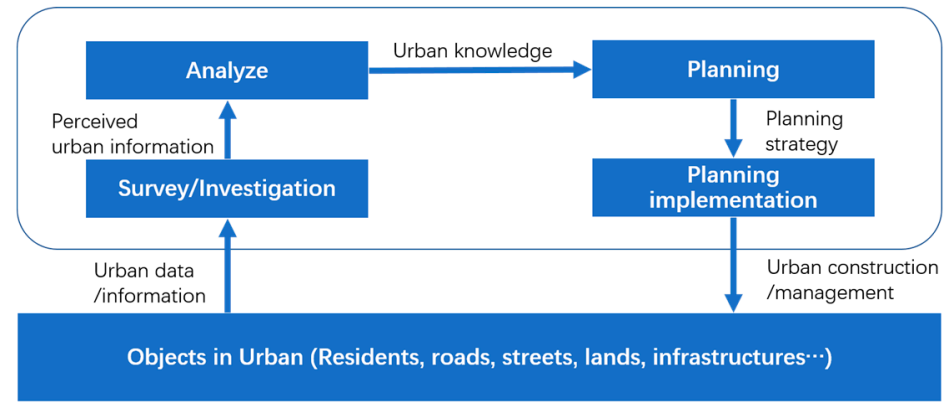

Figure 1.Information Eco-system Model of Urban Planning.

\subsection{Unbalance of the Ecological Chain-Lack of Human Information}

The basic information of cities can be divided to two types-unhuman information and human information. The former contains roads, lands, infrastructures, et al., those are changed relatively slowly. The latter is mainly focused on the activity and mobility of residents, those are changed very rapidly. It is very hard to track, measure and acquire human information. By the meantime, nowadays it is generally acknowledged that "human" has become the core of modern urban planning, instead of land or other entity objects. Modern urban projects which focused on residents' quality of life need more human information than ever.

Urban researchers have had to gain limited human information for decades because of limited sources, the disadvantaged technology and method. For example, the Chinese nationwide censes was taken every ten years. The data cannot precisely reflect the present situation. The outdated and inaccurate data make the planning quantitative analysis unscientific and improper.

\subsection{Solution}

For urban eco-system, optimizing information collection method is the key to achieve system balance. Using multisource data to analyze and measure human activity is one of the most important ways. Over past few years more and more urban researchers have started to use many kinds of big/open data to make more efficient quantitative analysis on the urban project. Long et al. use mobile phone signal data to measure the population density at the street level. The same author applied smart card data and taxi traces to measure human mobility and activity. These big/open data-based urban studies have proposed new methods and significantly improved the urban planning quantitative analysis. 
However, those data still cannot provide enough information to support quantitative analysis in micro scale. For example, the result of street population distribution via mobile phone data can't reflect the ground truth (Figure 2). Firstly it allocates all the people in a certain area into each street, which means people in the land are counted as ones on the streets but in fact they are not on the streets. Secondly it omits the group of people without mobile phone. Usually they could be kids under 10 and part of the aged. In micro scale, those deviations may lead to a bad or wrong conclusion.

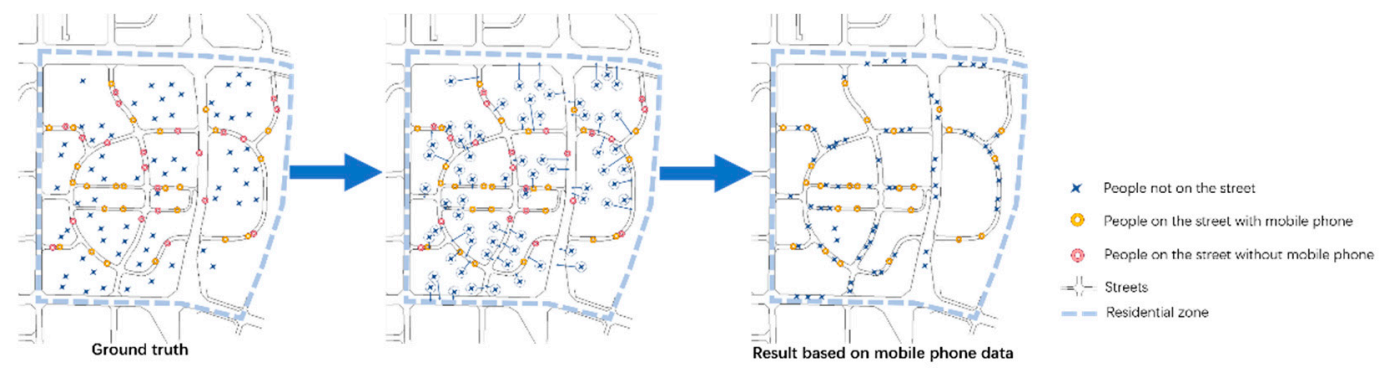

Figure 2.The flaws of mobile phone data in micro scale.

\section{Towards Information Ecosystem for Urban Planning-The Application of Video Data}

\subsection{A Proposed Scheme - Street Vibrancy Assessment based on Video Data}

Street Vibrancy is a typical urban planning element. It is an important topic which can measure the urban living quality. In street vibrancy quantitative research, in order to collect human mobility and activity information, some studies use field survey data and others use mobile phone data.

Video data on the other hand, has many advantages (Figure 3). Firstly, compared with the conventional data it can get continuous record for much longer time. Secondly, it directly records the ground truth instead of using indirect data to speculate, avoiding braised results. Thirdly, it can provide more accurate details about the survey target especially in micro scale. The multidimensional data could get a better description about human activity and mobility.
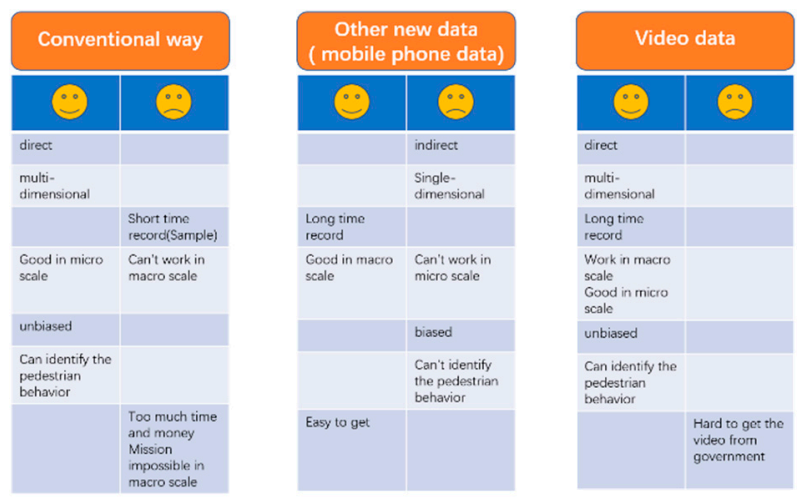

Figure 3.The compare of conventional way, other new data and video data.

Here we propose a scheme called "Street Vibrancy Assessment based on Video Data" (Figure 4). It has three steps. 


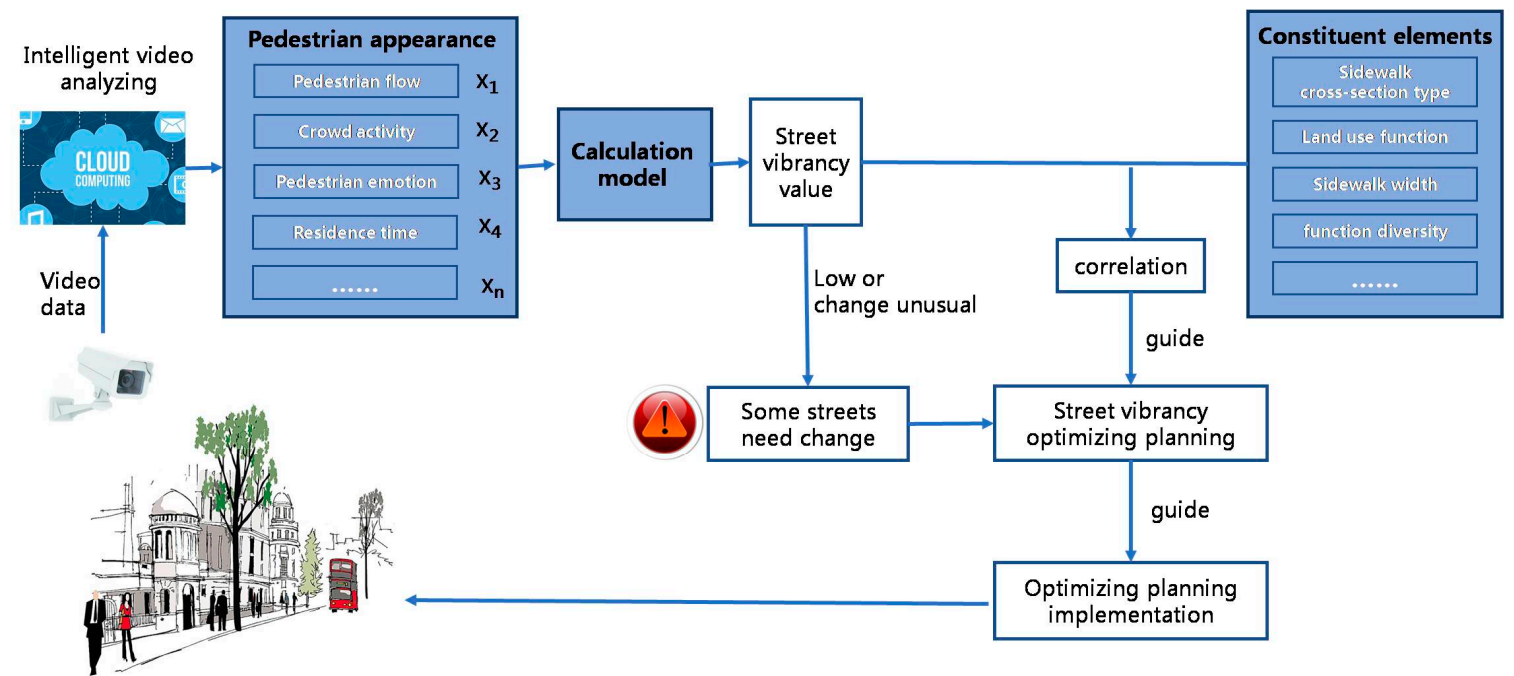

Figure4. The frame of street vibrancy assessment based on video data.

Step 1: The whole research is set around a residential zone which contains numbers of streets. Firstly more than one camera are put on each street. The video should record the whole situation of the street cross-section for at least one week. The video data is analyzed by intelligent video analyze technology to get the different kinds of information, such as pedestrian flow and activity and emotion, e.g.. Chosen information are put into a calculation formula to calculate a value to stand for street vibrancy.

Step 2: First of all, the street vibrancy value can be used to monitor the street. If compared with other similar ones the value is very low, or the value suddenly changes and the change is very unusual, it will give an automatic warning to remind the urban manager that maybe some streets need optimized.

On the other hand, each street has different constituent elements such as street cross-section type, sidewalk width, land use function, vehicle traffic situation et al. With the results above, the correlation could be found between the value and the constituent elements by regression analyze. The correlation will help the urban researcher to find out the main optimizing problems, to determine which one should be focused on, the cross-section or the function diversity or something else. Then, based on the basic information analyzing, strategies could be proposed after comprehensive analyzing.

Step 3: After the planning and the implementation has been done, the street vibrancy value should become better. Put the former value and the present value together, the implementation effectiveness of the street optimization could be charged.

\subsection{Implementation Detail and Discussion}

There are many kind of video analyzing technologies to achieve different kind of pedestrian appearances. In other word, the street vibrancy value $\mathrm{Y}$ can be calculated by:

$$
Y=f\left(x_{1}, x_{2}, \ldots, x_{n}\right)
$$

where $x_{i}$ stands for different pedestrian appearances with different attributes. The $f(*)$ is the function to define how different $x_{i}$ contribute to final value of $Y$, and it can be either linear or nonlinear with different parameters. It should be noticed that there is not always positive correlation between pedestrian appearance and the street vibrancy value. For example, the pedestrian flow indicates the account of people on the street, it is not "the more, the better". If somewhere are very crowded, like one pushing another, very hard to move, then it is obviously that the street is not a public space with sufficient vibrancy. 
All relative constituent elements should be considered as street vibrancy impact factors. Here we list some, sidewalk cross-section type, commercial type (land use function), function density, function diversity, sidewalk width, sidewalk length, vehicle traffic situation. For example, (1) According to different function parades, streets could be classified into 4 or more types: A-buildings (walls)-sidewalk-roadway, B-buildings (door open)-sidewalk-roadway, C-walls-sidewalk-roadway, D-walls-green bell-sidewalk-roadway.... (2) The commercial type of the stores right along the streets is also important, because a grocery, a fruit store, or a restaurant is totally different from a carwash.

Further analysis could be like below:

Similar streets should have similar vibrancy value on the time axis. If put all the streets vibrancy curve of one day together, find the unusual one/ones. Urban researcher could be reminded to find out the reason which makes the difference.

Compare two residential zones. Find out the difference by street vibrancy.

The results above can support many further research to guide the planning of enhancing street spatial quality, especially in micro scale, such as street walkability, quality of walking activity, street built environment.

Acknowledgments: This project was supported by the National Natural Science Foundation of China Grant (No. 61471248), the Fundamental Research Funds for the Central Universities (No.2015SCU04A11).

Author Contributions: L.H. proposed the basic idea, framework and drafted the article; L.Q. provided the main strategies for video analysis; X.H. helped for the application of Information Ecology; Y.P. completed the final approval of the version to be published.

Conflicts of Interest: No conflict of interest exits in the submission of this manuscript, and manuscript is approved by all authors for publication. I would like to declare on behalf of my co-authors that the work described was original research that has not been published previously, and not under consideration for publication elsewhere, in whole or in part. All the authors listed have approved the manuscript that is enclosed.

\section{References}

1. Herzallah, F.; Mukhtar, M. Organization Information Ecology and E-Commerce Adoption: Effect on Organizational SMEs Performance. Semin. Speech Lang. 2015, 11, 540-551.

2. Guan, J. Study on the Imbalance and Reconstruction of College English Classroom under Information Ecology. In Proceedings of the 4th International Education, Economics, Social Science, Arts, Sports and Management Engineering Conference, Yinchuan, China, 13-14 August 2016.

3. Long, Y.; Han, H.; Tu, Y.; Shu, X. Evaluating the effectiveness of urban growth boundaries using human mobility and activity records. Cities 2015, 46, 76-84.

4. Long, Y.; Zhou, Y. Quantitative Evaluation on Street Vibrancy and Its Impact Factors: A Case Study of Chengdu. New Archit. 2016, 1, 52-57.

5. Jacobs, J. The Death and Life of Great American Cities: The Failure of Town Planning; Penguin Books: London, UK, 1984.

6. Gehl, J.; Koch, J. Life between Buildings: Using Public Space; Van Nostrand Reinhold: New York, NY, USA, 1987.

(C) 2017 by the authors. Licensee MDPI, Basel, Switzerland. This article is an open access article distributed under the terms and conditions of the Creative Commons Attribution (CC BY) license (http://creativecommons.org/licenses/by/4.0/). 